\title{
EMPREENDEDORISMO E DESENVOLVIMENTO SOCIOECONÔMICO: ESTUDO COM MUNICÍPIOS DO ESTADO DE SÃO PAULO
}

\section{ENTREPRENEURSHIP AND SOCIOECONOMIC DEVELOPMENT: STUDY WITH CITIES OF THE STATE OF SÃO PAULO}

\author{
Rafael Toniolo da Rocha ${ }^{1}$ \\ Leandro Rodrigues ${ }^{2}$ \\ Simone Vasconcelos Ribeiro Galina ${ }^{3}$ \\ Elizabeth Krauter ${ }^{4}$
}

\begin{abstract}
Resumo
O desenvolvimento socioeconômico depende de muitas variáveis e o empreendedorismo é uma forma relevante de contribuir para seu avanço. No entanto, não há consenso internacional do impacto positivo do aumento do empreendedorismo no desenvolvimento socioeconômico local, além de existirem poucos estudos em países em desenvolvimento, como o Brasil. Assim, esta pesquisa teve como objetivo verificar a correlação entre empreendedorismo e desenvolvimento socioeconômico das principais cidades do estado de São Paulo (18 municípios) por meio da comparação da taxa de empreendedorismo (número de novos negócios por 1000 habitantes) com o Índice Firjan de Desenvolvimento Municipal (IFDM) no período de 2005 a 2013. Utilizaram-se dados secundários e a correlação foi a técnica de análise dos dados. Os resultados revelam existir correlação significativa entre as variáveis, mostrando que, apesar de encontradas correlações com indicadores de saúde e emprego e renda, educação é a variável mais fortemente relacionada com o avanço do empreendedorismo. Novos estudos devem ser realizados para explorar como o empreendedorismo influencia na qualidade de vida e no desenvolvimento socioeconômico dos municípios.
\end{abstract}

Palavras-chave: Empreendedorismo. Desenvolvimento socioeconômico. Índice Firjan de desenvolvimento municipal. Estado de São Paulo.

\footnotetext{
Abstract

Socioeconomic development depends on many variables and entrepreneurship is a relevant way of contributing to its progress. However, there is no international consensus on the positive impact of

1 Mestre em Administração de Organizações pela Universidade de São Paulo, Ribeirão Preto - SP, Brasil. E-mail: rafaeltoniolodarocha@gmail.com

${ }^{2}$ Doutorando em Administração de Organizações pela Universidade de São Paulo, Ribeirão Preto - SP, Brasil. E-mail: leandrorodrigues.sp@uol.com.br

${ }^{3}$ Doutora em Engenharia de Produção pela Universidade de São Paulo. Professora da Universidade de São Paulo, Ribeirão Preto - SP, Brasil. E-mail: svgalina@usp.br

4 Doutora em Administração (USP). Professora da Universidade de São Paulo, Ribeirão Preto - SP, Brasil. E-mail: ekrauter@usp.br
} 
increased entrepreneurship on local socioeconomic development, and there are few studies in developing countries, such as Brazil. Thus, this research aimed to verify the correlation between entrepreneurship and socioeconomic development of the main cities in the state of São Paulo (18 municipalities) by comparing the rate of entrepreneurship (number of new businesses per 1000 inhabitants) with the Firjan Municipal Development Index (IFDM) from 2005 to 2013. Secondary data were used and the correlation was the data analysis technique. The results show that there is a significant correlation between the variables, demonstrating that although correlations were found with health, and employment and income indicators, education is the variable most strongly related to the advance of entrepreneurship. New studies should be carried out to explore how entrepreneurship influences the quality of life and socioeconomic development of municipalities.

Keywords: Entrepreneurship. Socioeconomic development. Firjan Municipal Development Index. State of São Paulo.

\section{Introdução}

Muitos estudos têm sido realizados com o intuito de relacionar a atividade empreendedora e o desenvolvimento econômico. Há de se considerar que o empreendedorismo desempenha um papel importante no desenvolvimento, podendo ser considerado um elemento fundamental para o desenvolvimento econômico dos países (SCHUMPETER, 1997).

Vários pesquisadores têm corroborado a teoria de Schumpeter, no sentido de apresentar resultados que comprovem a ligação do desenvolvimento econômico e o empreendedorismo. Estes resultados podem ser observados, por exemplo, nos trabalhos de Audretsch, Belitski e Desai (2015), Audrestch, Keilbach e Lehmann (2006) e Adusei (2016). Outros autores encontraram resultados positivos quando relacionaram empreendedorismo e emprego, ou seja, a atividade empreendedora pode colaborar com o aumento da taxa de empregos no longo prazo, de acordo com Batista, Escaria e Madruga (2008), Chen (2014) e Kasseeah (2016).

Ao discutir esse tema, é importante considerar também os fatores que podem colaborar com a atividade empreendedora. Dentre esses fatores, a educação da população é um ponto chave para o desenvolvimento do empreendedorismo (AUDRETSCH; BELITSKI; DESAI, 2015; GARCIA, 2014).

No contexto brasileiro, foram identificados trabalhos que apresentam estudo empírico que busque explicar a relação entre as duas variáveis, de forma que dois trabalhos (FONTENELE, 2010; SOUZA; LOPES JUNIOR, 2011) buscam essa relação em nível de país e outros dois (FELIX; FARAH JUNIOR, 2013; CANEVER et al., 2010) realizando estudos em um nível regional (estadual e municipal). Esses dados corroboram o apontamento de Bruton, Ahlstrom e Obloj (2008) ao relatar que grande parte das pesquisas referentes ao empreendedorismo e desenvolvimento estão relacionadas a países desenvolvidos, principalmente, Europa e Estados Unidos, enquanto existe uma carência desses estudos em economias em desenvolvimento, como Brasil, Índia e Rússia, havendo uma lacuna maior a ser preenchida no estudo da relação do empreendedorismo e desenvolvimento socioeconômico nesses países.

Estudos realizados no Brasil em nível regional observaram que o empreendedorismo foi realmente um fator importante no desenvolvimento econômico daquelas localidades, além de colaborar também com o desenvolvimento humano da população (FELIX; FARAH JUNIOR, 2013; CANEVER ET AL, 2010). Entretanto, ambos os estudos foram realizados em estados da região Sul do país, Paraná e Rio Grande Sul, e dada a dimensão continental e as diferenças regionais do Brasil, não é possível afirmar que o mesmo efeito positivo será encontrado em outros estados e regiões do país. Assim, pode-se identificar a escassez de trabalhos, no Brasil, que considerem o estudo da relação entre o empreendedorismo e o desenvolvimento local.

Ao vislumbrar essa oportunidade de pesquisa, este trabalho tem por objetivo contribuir com o preenchimento desta lacuna ao verificar a correlação entre o empreendedorismo e o desenvolvimento socioeconômico das principais cidades do estado de São Paulo. Esse objetivo é atingido por meio de um indicador para a taxa de empreendedorismo.

Portanto, a relevância deste trabalho justifica-se pelo fato de que avaliar empreendedorismo é importante para desenvolvimento regional, há um gap a ser preenchido quando se refere a estudos 
relacionando empreendedorismo e desenvolvimento econômico em países em desenvolvimento por meio de um indicador para que seja possível comparar o nível de empreendedorismo em cidades de portes diferentes.

Além do avanço para o conhecimento na área de empreendedorismo e desenvolvimento regional, esta pesquisa contribui, também, com a geração de informações relevantes para a administração dos municípios, colaborando com a gestão pública por meio de indicadores que auxiliem na tomada de decisão e nas alocações dos recursos públicos, uma vez que não existem muitas informações sobre empreendedorismo disponíveis em nível municipal.

A seguir será apresentado o referencial teórico, que versará sobre a relação entre empreendedorismo e desenvolvimento socioeconômico. Depois, será demonstrada a metodologia, os resultados e discussões e, por fim, as considerações finais.

\section{Empreendedorismo e desenvolvimento}

Schumpeter (1997) apresentava o empreendedorismo como um "fenômeno fundamental" para o desenvolvimento econômico. No entanto, alguns autores têm considerado que o empreendedorismo pode impedir o crescimento econômico, podendo inclusive acentuar a pobreza e a desigualdade (HE; CHEN, 2016).

Para sustentar as ideias de Schumpeter (1997), estudos empíricos têm surgido com o intuito de demonstrar a ligação entre a atividade empreendedora e o desenvolvimento econômico (FRITSCH; MULLER, 2004; TOMA; GRIGORE; MARINESCU, 2014).

Importante destacar que, por meio dos estudos de Audretsch, Belitski e Desai (2015), Baptista, Escaria e Madruga (2008) e Garcia (2014), observou-se que alguns elementos são constantes na relação entre desenvolvimento socioeconômico e empreendedorismo. Esses autores apontam os elementos educação, emprego e renda e saúde como relevantes.

O estudo de Audretsch, Belitski e Desai (2015), por exemplo, corrobora a relação entre empreendedorismo e desenvolvimento socioeconômico. Os autores identificaram, por meio de estudos com pequenas, médias e grandes cidades europeias, evidências de que o empreendedorismo apresenta forte e imediato impacto sobre o desenvolvimento econômico urbano, ou seja, existe uma evidente e convincente ligação entre a atividade empreendedora e a performance econômica (AUDRESTCH; KEILBACH; LEHMANN, 2006)

Apesar da relação encontrada pelos autores, foram identificadas diferenças no impacto, quando considerado o tamanho das cidades, quanto aos efeitos diretos referentes à taxa de emprego e aos efeitos indiretos: eficiência, inovação, variedade e competitividade. Os efeitos diretos foram encontrados em todas as cidades, não importando o tamanho, enquanto o impacto sobre os efeitos indiretos não foi observado nas pequenas e médias cidades (AUDRETSCH; BELITSKI; DESAI, 2015).

Sobre o elemento de emprego e renda, que está associado à geração de empregos, Baptista, Escaria e Madruga (2008), em pesquisa realizada em Portugal, evidenciam efeitos positivos do empreendedorismo sobre a criação de empregos. Esses efeitos foram observados em três períodos, onde a startups em seu início apresenta fraca influência sobre a taxa de emprego, num segundo período apresenta um efeito negativo somente após o oitavo ano, terceiro período, começa a apresentar um efeito positivo.

Resultados semelhantes foram encontrados por Mueller, van Stel e Storey (2008) na GrãBretanha, considerando que os efeitos sobre a taxa de emprego acontece em três fases, ou seja, de acordo com Fritsch e Mueller (2004) as startups são capazes de colaborar com o crescimento da taxa de emprego somente a partir de um longo prazo.

Dvouletý (2017), através de sua pesquisa nas regiões da República Tcheca, observou que as taxas de empreendedorismo estão relacionadas com baixos índices de desemprego. Argumenta, também, que as altas taxas de criação de novos negócios ou parcerias foram associadas com altos níveis de renda per capita. Importante destacar que as regiões onde foram encontradas altas taxas de renda per capita foram também aquelas onde foi observado o maior número de pessoas com níveis mais altos de escolaridade.

Chen (2014) e Kasseeah (2016) também encontraram resultados que confirmam a relação entre renda per capita e a intensidade de novos negócios, existindo uma relação positiva entre empreendedorismo e desenvolvimento econômico e, consequentemente, surgimento de novos postos de trabalho. Para Kasseeah (2016) os resultados confirmam que o empreendedorismo é um fator 
importante para o desenvolvimento econômico dos países independente do seu nível de desenvolvimento.

Essa realidade vai ao encontro dos fatores que Audretsch, Belitski e Desai (2015) identificaram como relevantes para os efeitos do empreendedorismo no desenvolvimento das cidades. A presença de centros de desenvolvimentos e pesquisas nas grandes cidades, acompanhado de uma mão de obra mais sofisticada devido à presença de universidades podem ser considerados fatores importantes para o impacto do empreendedorismo sobre os efeitos indiretos (eficiência, inovação, variedade e competitividade) nas cidades maiores (AUDRETSCH; BELITSKI; DESAI, 2015).

Garcia (2014), em sua pesquisa envolvendo 184 cidades em vinte países europeus, encontrou resultados empíricos de que aquelas cidades onde o número de pessoas com nível superior de escolaridade era maior, apresentaram taxas mais elevadas de atividade empreendedora. Concluiu também, que, havia uma ligação positiva entre o empreendedorismo, o tamanho da cidade e o autoemprego.

Em pesquisa realizada na Alemanha observou-se que nas regióes onde se encontram centros universitários com grande capacidade de gerar e compartilhar conhecimento também foi possível encontrar um número elevado de startups, principalmente ligadas ao setor tecnológico (AUDRESTCH; KEILBACH; LEHMANN, 2006).

Ainda dentro da perspectiva do desenvolvimento econômico proporcionado pelo empreendedorismo, Souza e Lopes Junior (2011) verificaram que existe uma correlação negativa entre o indicador de empreendedorismo Total Entrepreneurship Activity (TEA) divulgado pelo Global Entrepreneurship Monitor (GEM) e o Índice de Desenvolvimento Humano (IDH).

Nos países onde o IDH é baixo observou-se um TEA elevado, ou seja, nos países com baixo desenvolvimento humano existe um alto índice de empreendedorismo e nos países com alto IDH foi observada uma baixa atividade empreendedora (SOUZA; LOPES JUNIOR, 2011). Esse fenômeno também foi considerado por Acs, Desai e Hessels (2008) e Prieger et al. (2016).

Evidências do impacto positivo do empreendedorismo sobre desenvolvimento econômico também foi identificado em pesquisas no contexto chinês (LI; DING, 2015; HE; CHEN, 2016). He e Chen (2016), que utilizaram o IDH como uma das métricas, encontraram resultados que sugerem que aquelas regiões com maiores atividades empreendedoras são aquelas capazes de absorver toda a mão de obra advindas das áreas rurais e que apresentam população com maior qualidade de vida.

Nessas regiões observa-se, ainda, melhor qualificação da mão-de-obra, crescimento da atividade empreendedora, melhores condições de saúde e menor quantidade de pessoas vivendo abaixo da linha de pobreza. Por outro lado, devido ao crescimento industrial, a região apresenta maior emissão de $\mathrm{CO}_{2}$, quando comparada com outras regiões chinesas (HE; CHEN, 2016).

Em outro estudo chinês, que teve como finalidade a investigação dos impactos da atividade empreendedora e da inovação na redução da pobreza, verificou-se efeito positivo do empreendedorismo na redução da pobreza (SI et al., 2015).

Esses autores identificaram que os novos negócios criados pelos camponeses, acompanhados de projetos inovadores, foram responsáveis por tirar aquela região de uma pobreza extrema. Esse fato transformou a região na mais empreendedora da China, além de colaborar com a diminuição da pobreza em outros países, uma vez que grande número de estrangeiros buscam a região para estudar ou desenvolver novos negócios para seus países de origem (SI et al., 2015).

Um fator que interfere nesta relação entre empreendedorismo e desenvolvimento socioeconômico é tipo de empreendedorismo. Os países com maior renda per capita apresentam maior índice de empreendedorismo por oportunidade enquanto aqueles com menor renda per capita apresentam uma alta taxa de empreendedorismo por necessidade (ACS; DESAI; HESSELS, 2008; FONTENELE, 2010). No caso do Brasil, predomina o empreendedorismo por necessidade (ACS; DESAI; HESSELS, 2008).

Dentro dessa perspectiva do tipo de empreendedorismo, Amoros, Borraz e Veiga (2016) demonstraram por meio de um estudo envolvendo os países da América Latina, que a taxa de empreendedorismo por necessidade nessa região não está relacionada com a riqueza dos países, mas aos ciclos econômicos, ou seja, quanto maior o crescimento econômico maior a taxa de empreendedorismo, corroborando os conceitos de Li e Ding (2015) e Chen (2014) ao evidenciarem que os impactos do empreendedorismo podem variar em diferentes períodos econômicos.

Ainda de acordo com Amoros, Borraz e Veiga (2016), os resultados não confirmam a relação entre empreendedorismo por necessidade e os níveis de desemprego na região, como propõe Acs, 
Desai e Hessels (2008) e Fontenele (2010). Amoros, Borraz e Veiga (2016) encontraram as mesmas características para os dois tipos de empreendedorismo (oportunidade e necessidade). A pesquisa demonstrou que nas realidades de altas taxas de desemprego, os profissionais mais qualificados não se sentem atraídos pelos salários, que estão no seu nível mais baixo, o que faz com que optem por abrirem seus próprios negócios.

Outro resultado observado pelos autores é que o crescimento econômico está relacionado com o empreendedorismo por oportunidade, além de resultados que sugerem uma relação positiva entre empreendedorismo por necessidade e inflação e, ainda, o grau de informalidade da economia (AMOROS; BORRAZ; VEIGA, 2016).

O estudo sobre empreendedorismo em diferentes regióes de diversos países da Europa, levou Bosma e Sternberg (2014) argumentarem que a diversidade econômica afeta a ocorrência de novos negócios, demonstrando que não haver diferença na taxa de empreendedorismo entre as principais cidades e as outras regiões do país. Isso contradiz a hipótese de Audretsch, Belitski e Desai (2015), em que as maiores áreas urbanas levam vantagem em relação às menores, quanto ao nível de atividade empreendedora.

Bosma e Sternberg (2014), evidenciam que as regiões com maior PIB favorecem a criação de novos negócios por oportunidade, enquanto que o aumento no desemprego leva ao aparecimento de novos negócios por necessidade.

Sobre o impacto da variável saúde na relação de empreendedorismo e desenvolvimento socioeconômico, Frederick e Monsen (2011), a partir de pesquisas sobre o empreendedorismo e investimento públicos em saúde na Nova Zelândia, identificaram que o empreendedorismo prospera em ambientes com baixas situações de bem-estar social. Para eles, variações nos investimentos públicos em saúde podem trazer estímulo à atividade empreendedora.

Os autores destacam que em países desenvolvidos e com altos salários, os investimentos públicos em saúde possuem uma relação negativa com a taxa de atividade empreendedora, enquanto que os gastos com saúde privada colaboram com o aumento da taxa de empreendedorismo.

Um fator que pode colaborar com o a criação de novos negócios é a cultura empreendedora da população. Fritsch e Wyrwich (2017) demonstram por meio de resultados empíricos que as taxas de empreendedorismo são maiores em algumas regiões da Alemanha.

Essas regiões apresentam em suas raízes uma cultura empreendedora que permaneceu ativa ao longo do tempo, mesmo devido às dificuldades e instabilidades financeiras e sociais enfrentadas pela região. Também são as mesmas regiões que apresentaram altas taxas de autoemprego em 1925 e, atualmente, contam com alta taxa de criação de novos negócios. Os autores argumentam que a criação de uma cultura empreendedora é um investimento de longo prazo (FRITSCH; WYRWICH, 2017).

Conforme demonstrado até aqui, grande parte das pesquisas referentes ao empreendedorismo relaciona-se aos países da Europa e Estados Unidos, enquanto estudos fora dessas regiões ainda são um tanto limitados, principalmente nas economias em desenvolvimento (BRUTON; AHLSTROM; OBLOJ, 2008).

Nesse sentido, Adusei (2016) realizou um estudo em doze países africanos a fim de observar se o empreendedorismo era capaz de promover o crescimento econômico naquela região. A partir dos resultados obtidos, concluiu que, realmente, o empreendedorismo tem sustentado o crescimento econômico naqueles países.

No Brasil, algumas pesquisas têm sido realizadas no sentido de relacionar a atividade empreendedora e desenvolvimento econômico. Felix e Farah Junior (2013), ao realizarem um estudo em municípios do estado do Paraná, região do sul do Brasil, evidenciaram que existe uma correlação positiva entre o empreendedorismo e o desenvolvimento dessas localidades, considerando um índice que demonstra o nível de ambiente favorável de um local para as micro e pequenas empresas e um outro indicador de emprego e renda.

De acordo com Canever et al (2010), em pesquisa realizada em outro estado do sul do país, Rio Grande do Sul, concluíram que existe também uma forte relação entre empreendedorismo e desenvolvimento econômico e humano. Dentre outros resultados interessantes, demonstram que o nível de educação da população desempenha um papel importante no desenvolvimento de novos negócios.

Dessa forma, uma vez constatada a relação entre empreendedorismo e desenvolvimento socioeconômico em vários estudos, Prieger et al. (2016) fazem o seguinte questionamento: apesar da alta taxa de empreendedorismo, por que muitos países em desenvolvimento continuam pobres? $\mathrm{E}$ 
propõe três fatores alternativas de resposta: esses países necessitam de mais empreendedorismo, devem existir outros fatores que reduzem o impacto do empreendedorismo sobre o desenvolvimento (como a falta de pesquisa e desenvolvimento e de empresas de venture capital) ou talvez o tipo de empreendedorismo não é o mais apropriado (PRIEGER et al., 2016).

\section{Metodologia}

Trata-se de um estudo quantitativo com dados secundários. Este trabalho foi composto de duas etapas: coleta e construção do banco de dados e análise dos dados. A seguir são apresentadas as variáveis do estudo, a forma de coleta e a construção do banco de dados, o universo amostral e por fim, a forma de análise dos dados.

\section{Variáveis da pesquisa}

Muitas pesquisas têm sido realizadas no sentido de aprofundar o conhecimento a respeito dos fatores que estimulam a atividade empreendedora tanto a nível regional ou de cidades (GARCIA, 2014).

Pesquisadores têm utilizado vários indicadores para estudar a taxa de empreendedorismo, pois, na verdade, não existe uma unanimidade entre eles a respeito de uma medida ideal para tal estudo (ADESUI, 2016). Muitos têm utilizado a taxa de empreendedorismo fornecida pelo Global Entrepreneurship Monitor (GEM), que é uma pesquisa realizada em vários países ao redor do mundo a fim de identificar o grau de atividade empreendedora (ACS; DESAI; HESSELS, 2008; FONTENELE, 2010; SOUZA; LOPES JUNIOR, 2011; PRIEGER ET AL, 2016).

Outros estudos utilizam ainda o Índice de Desenvolvimento Humano (IDH) (SOUZA; LOPES JUNIOR, 2011, HE; CHEN, 2016), bem como o Produto Interno Bruto (PIB) a fim de apresentar a relação do empreendedorismo e o desenvolvimento (AUDRESTCHI; BELITSKI; DESAI, 2015, ADUSEI, 2016) ou ainda a taxa de emprego local, no sentido de correlacionar o empreendedorismo e a criação de emprego (BAPTISTA; ESCARIA; MADRUGA, 2008).

Alguns outros indicadores também são utilizados, como por exemplo, o Eurostat Urban Audit que fornece informações e métricas padronizadas relacionados a vários aspectos relacionados à qualidade de vida nas cidades europeias (GARCIA, 2014; DVOULETÝ, 2017). Em outros estudos foram considerados o número de empresas registradas dentro do ano fiscal no qual a pesquisa foi realizada (ADUSEI, 2016; GARCIA, 2014, BAPTISTA; ESCARIA; MADRUGA, 2008, KASSEEAH, 2016; DVOULETÝ, 2017).

Em pesquisa realizada por Felix e Farah Junior (2013), também com a finalidade de correlacionar empreendedorismo e o desenvolvimento em determinadas localidades de um estado do sul do país, utilizaram-se os indicadores disponibilizados pela Federação das Indústrias do Rio de Janeiro (FIRJAN) e pelo Serviço de Apoio às Micros e Pequenas Empresas do Estado do Paraná (SEBRAE Paraná).

Para atender ao propósito de verificar possíveis correlações entre o desenvolvimento local e empreendedorismo foi adotado como variáveis a taxa de empreendedorismo bruto - TEB - $\left(\mathrm{n}^{0}\right.$ de novos negócios criados em um período $\mathrm{n}$ a cada 1000 habitantes) e o índice Firjan de Desenvolvimento Municipal (IFDM). A taxa de empreendedorismo bruto (TEB) foi desenvolvida com o intuito de tornar comparável municípios que apresentem diferentes portes. Ela teve como inspiração a taxa de empreendedorismo (TEA) fornecida pelo Global Entrepreneurship Monitor (GEM), mas considera também o contingente populacional, sendo possível visualizar o montante de empresas que iniciam suas atividades num determinado período para cada 1000 habitantes. Essa taxa auxilia em análises que visem a investigar o papel das organizações no desenvolvimento socioeconômico de uma região.

O índice TEB foi adaptado a partir dos estudos de Dvouletý (2017) que utilizou o taxa de criação de novos negócios e parcerias per capita como métrica da atividade empreendedora nas diversas regiões da República Tcheca onde foi realizada a pesquisa; de Kasseeah (2016) que também utilizou a taxa de novas empresas registradas dividido por grupos de mil pessoas, com idade de 15 a 64 anos e por fim de Canever et al (2010) que observaram o número de estabelecimento de novas empresas a cada 1000 habitantes de diferentes países.

Para a escolha desse índice levou-se em consideração a importância da relação entre crescimento populacional e criação de novos negócios, conforme argumenta Canever et al. 2010. 
O IFDM - Índice FIRJAN de Desenvolvimento Municipal - é um estudo do Sistema FIRJAN que acompanha anualmente o desenvolvimento socioeconômico de todos os mais de 5 mil municípios brasileiros em três áreas de atuação: Emprego \& renda, Educação e Saúde. Criado em 2008, ele é feito, exclusivamente, com base em estatísticas públicas oficiais, disponibilizadas pelos ministérios do Trabalho, Educação e Saúde (FIRJAN, 2017). Esse índice foi inspirado no IDH - índice de Desenvolvimento Humano da ONU, considerando emprego e Renda, educação e saúde, mais faz uma análise mais profunda considerando também outras variáveis, como é demostrado no quadro 1 . 0 índice varia de 0 (mínimo) a 1 ponto (máximo) para classificar o nível de cada localidade em quatro categorias: baixo (de 0 a 0,4$)$, regular $(0,4$ a 0,6$)$, moderado (de 0,6 a 0,8$)$ e alto $(0,8$ a 1 ) desenvolvimento, ou seja, quanto mais próximo de 1 , maior o desenvolvimento da localidade.

Além das variáveis TEB e IFDM, foram utilizadas também 3 variáveis de controle: educação, saúde e emprego e renda. Todas essas variáveis foram obtidas por meio sistema FIRJAN, assim como foi obtido o IFDM. Elas compõem o IFDM e são divulgadas também para cada área que compõe o índice. Os componentes de cada uma das variáveis de controle são demonstrados no Quadro 1.

Outra variável gerada pelo estudo foi composta pelas taxas de crescimento dos indicadores TEB, IFDM e o crescimento demográfico, no período de 2005 a 2013, para que fosse possível observar o comportamento de cada indicador em cada uma das cidades no período estudado.

Quadro 1: Componentes do IFDM por área de desenvolvimento

\section{IFDM}

\begin{tabular}{|c|c|c|}
\hline Emprego\&Renda & Educação & Saúde \\
\hline $\begin{array}{l}\text { - Geração de emprego formal } \\
\text { - Absorção da mão de obra local } \\
\text { - Geração de Renda formal } \\
\text { - Salários médios do emprego } \\
\text { formal } \\
\text { - Desigualdade }\end{array}$ & $\begin{array}{l}\text { - Matrículas na educação infantil } \\
\text { - Abandono no ensino } \\
\text { fundamental } \\
\text { - Distorção idade-série no ensino } \\
\text { fundamental } \\
\text { - Docentes com ensino superior } \\
\text { no ensino fundamental } \\
\text { - Média de horas aula diárias no } \\
\text { ensino fundamental } \\
\text { - Resultado do IDEB no ensino } \\
\text { fundamental }\end{array}$ & $\begin{array}{l}\text { - Número de consultas pré-natal } \\
\text { - Óbitos por causas mal definidas } \\
\text { - Óbitos infantis por causas } \\
\text { evitáveis } \\
\text { - Internação sensivel à atenção } \\
\text { básica (ISAB) }\end{array}$ \\
\hline
\end{tabular}

Fonte: FIRJAN (2015)

\section{Coleta e construção do banco de dados}

Vale ressaltar que no Brasil não há indicadores que gerem essa informação. Para construir a Taxa de Empreendedorismo Bruto (TEB) foram utilizadas as informações disponíveis na Junta Comercial do Estado de São Paulo (JUCESP), que reúne todos os registros de empresas ativas e desativas. A série histórica da população dos municípios objeto de estudo foram obtidos por meio da Fundação Sistema Estadual de Análise de Dados (SEADE) que reúne dados de todos municípios do estado de São Paulo.

Garcia (2014) destaca que a vantagem de se considerar a pesquisa em limites regionais é a ênfase na responsabilidade política, ou seja, cada indicador está sob a responsabilidade das autoridades locais. Sendo assim, limites administrativos podem otimizar o nível da análise pelo fato das informações serem mais acessíveis, além de que os indicadores podem ser adaptados aos contextos locais. 


\section{Universo amostral}

Foram objeto de estudo os municípios-sede das Regiões Administrativas e das Aglomerações Urbanas do estado de São Paulo, totalizando 18 municípios estudados. A escolha dessas cidades se deve ao fato de representarem importantes polos regionais de desenvolvimento no estado. Os municípios são: Araçatuba, Araraquara, Barretos, Bauru, Campinas, Franca, Jundiaí, Marília, Piracicaba, Presidente Prudente, Registro, Ribeirão Preto, Santos, São Carlos, São José do Rio Preto, São José dos Campos, São Paulo e Sorocaba.

O período abrangido para coleta e análise dos dados foi de 2005 a 2013 pelo fato de compreender a totalidade dos índices divulgados pela Firjan.

\section{Análise dos dados}

Para a análise de dados foi utilizada a correlação de Pearson, que nos mostra o grau de relacionamento entre duas variáveis. Nesse caso, existe um relacionamento positivo perfeito quando o valor for igual a 1, positivo moderado quando estiver em torno de 0,70 e haverá ausência de relacionamento quando for igual a 0 . Esse relacionamento pode ser também negativo moderado 0,70 e negativo perfeito, quando for igual a -1 (STEVENSON, 2001).

Para a realização das análises foi construído um banco de dados com todas as informações extraídas do portal da JUCESP, do sistema FIRJAN e do SEADE para o período estudado. Todas as análises estatísticas foram realizadas por meio do Excel.

\section{Resultados e discussão}

O banco de dados construído para esta pesquisa permite realizar uma análise histórica dos 18 municípios objetos de estudo, no que se refere à evolução no número de empresas registradas na JUCESP, a taxa de mortalidade anual, a composição por porte das empresas nascentes, a variação demográfica, a variação do índice Firjan de Desenvolvimento Municipal e suas respectivas subáreas (educação, saúde e emprego e renda).

Os resultados evidenciados na Tabela 1, apresentam um ranking de crescimento da taxa de empreendedorismo bruto (TEB) ao longo do período analisado, além de trazerem o crescimento do IFDM e o crescimento demográfico para o mesmo período, observando-se a população em 2013. Esses dados são reunidos para demonstrar que a taxa de empreendedorismo independe do porte do município, diferente do que conclui Garcia (2004) em seu estudo em cidades europeias ao demonstrar uma relação positiva entre empreendedorismo, o porte da cidade e o auto emprego. $\mathrm{O}$ município mais populoso da amostra, São Paulo, apresentou o $3^{\circ}$ maior crescimento (205\%) enquanto que o menor município, Registro, apresentou o maior crescimento no período (303\%).

A relação entre o crescimento da taxa de empreendedorismo e o crescimento do índice Firjan de Desenvolvimento Municipal para esse período não apresentou nenhum padrão. Santos apresentou um dos maiores crescimentos no IFDM (12,15\%), mas foi o que apresentou um dos menores crescimentos da TEB também (128\%). Araraquara apresentou o menor crescimento no IFDM $(1,01 \%)$ e um crescimento de $149 \%$ da TEB. Registro apresentou o maior crescimento da TEB (303\%) e o maior desenvolvimento socioeconômico $(15,04 \%)$, além de ser a única cidade a compor a amostra que possui uma população inferior a 100 mil habitantes e ser a única a apresentar um decrescimento demográfico no período. 
Tabela 1: Crescimento TEB x Crescimento IFDM x Crescimento demográfico (período 2005-2013)

\begin{tabular}{llllll}
\hline \hline Posição & Município & $\begin{array}{l}\text { Crescimento } \\
\text { TEB }\end{array}$ & $\begin{array}{l}\text { Crescimento } \\
\text { IFDM }\end{array}$ & $\begin{array}{l}\text { Crescimento } \\
\text { demográfico }\end{array}$ & $\begin{array}{l}\text { População } \\
2013^{*}\end{array}$ \\
\hline $1^{\circ}$ & Registro & $303 \%$ & $15,04 \%$ & $-0,6 \%$ & 54.107 \\
$2^{\circ}$ & Sorocaba & $226 \%$ & $5,36 \%$ & $12,6 \%$ & 608.269 \\
& & & & & 11.446 .27 \\
$3^{\circ}$ & São Paulo & $205 \%$ & $8,59 \%$ & $5,3 \%$ & 5 \\
$4^{\circ}$ & Campinas & $201 \%$ & $3,26 \%$ & $8,6 \%$ & 1.112 .050 \\
$5^{\circ}$ & Pres. Prudente & $192 \%$ & $8,60 \%$ & $6,5 \%$ & 211.832 \\
$6^{\circ}$ & Marília & $188 \%$ & $7,19 \%$ & $6,7 \%$ & 221.378 \\
$7^{\circ}$ & São Carlos & $185 \%$ & $5,62 \%$ & $10,2 \%$ & 228.556 \\
$8^{\circ}$ & Ribeirão Preto & $183 \%$ & $4,65 \%$ & $13,5 \%$ & 629.855 \\
$9^{\circ}$ & Bauru & $182 \%$ & $1,20 \%$ & $5,9 \%$ & 350.392 \\
$10^{\circ}$ & SJ dos Campos & $176 \%$ & $2,67 \%$ & $12,1 \%$ & 654.827 \\
$11^{\circ}$ & Jundiaí & $165 \%$ & $5,91 \%$ & $10,3 \%$ & 382.363 \\
$12^{\circ}$ & Piracicaba & $152 \%$ & $12,89 \%$ & $7,3 \%$ & 372.553 \\
$13^{\circ}$ & Barretos & $152 \%$ & $5,33 \%$ & $5,7 \%$ & 114.027 \\
$14^{\circ}$ & Araraquara & $149 \%$ & $1,01 \%$ & $10,1 \%$ & 215.080 \\
$15^{\circ}$ & Franca & $134 \%$ & $13,32 \%$ & $7,3 \%$ & 326.042 \\
$16^{\circ}$ & Araçatuba & $131 \%$ & $6,14 \%$ & $4,9 \%$ & 184.491 \\
$17^{\circ}$ & Santos & $128 \%$ & $12,15 \%$ & $0,5 \%$ & 421.896 \\
$18^{\circ}$ & SJ do Rio Preto & $115 \%$ & $6,86 \%$ & $9,9 \%$ & 421.169 \\
\hline- & Média & $171 \%$ & $6,99 \%$ & $7,6 \%$ & - \\
\hline
\end{tabular}

Fonte: dados da pesquisa. *SEADE (2017).

$\mathrm{O}$ crescimento demográfico não se mostrou determinante para taxa de crescimento. Registro obteve um crescimento demográfico negativo $(-0,6 \%)$ e apresentou o maior crescimento da TEB enquanto que Santos, também com o crescimento demográfico pouco significativo $(0,5 \%)$, apresentou um dos menores crescimento na TEB (128\%). O mesmo se pode observar nos municípios com maior crescimento demográfico: Sorocaba $(12,6 \%)$ apresentou um dos maiores crescimento de novas empresas proporcionalmente a sua população (226\%) enquanto que Ribeirão Preto e São José dos Campos, municípios do mesmo porte e crescimento demográfico semelhante, $13,5 \%$ e $12,1 \%$ respectivamente, acumularam um crescimento na taxa de empreendedorismo bruto menor, $183 \% \mathrm{e}$ $176 \%$ respectivamente.

Para a análise da correlação entre o empreendedorismo e o desenvolvimento socioeconômico foi utilizado o IFDM e cada um dos seus componentes isoladamente, educação, saúde e emprego e renda, como é demonstrado na Tabela 2. Os coeficientes destacados em azul representam correlação positiva superior a 0,7 e os coeficientes destacados em vermelho demonstram as correlações negativas.

A Tabela 2 reúne na segunda coluna as correlações entre a TEB e o IFDM, que representam o empreendedorismo e o desenvolvimento socioeconômico, respectivamente, e na terceira, quarta e última coluna, a correlação entre o empreendedorismo (TEB) e cada uma das variáveis, separadamente: Educação, Saúde e Emprego e Renda, respectivamente.

Diferente do que concluíram Souza e Lopes Júnior (2011), o resultado da correlação entre empreendedorismo e desenvolvimento socioeconômico na maioria das cidades mostrou-se relevante $(>0,70)$ corroborando os estudos realizados por Schumpeter (1997); Toma, Grigore e Marinescu (2014) e Audretsch, Belitski e Desai (2015).

A correlação positiva encontrada na maioria das cidades paulistas (com exceção de Araraquara e São José dos Campos), objeto deste estudo, teve resultado similar ao encontrado por Felix e Farah Júnior (2013) ao realizar estudo visando a relacionar empreendedorismo e desenvolvimento socioeconômico em cidades do estado do Paraná e também com os resultados obtidos por Canever et al. (2010) em estudo realizado em cidades do Rio Grande do Sul.

Outra variável que se mostrou significativamente relacionada ao aumento das empresas nascentes e ao desenvolvimento socioeconômico foi a educação. Essa variável foi a única que foi positiva em todos os municípios estudados e a correlação entre a taxa de empreendedorismo e o 
índice Firjan Educação foi positivo e superior a 0,7 em 95\% dos municípios (apenas Araçatuba apresentou correlação inferior a 0,7 ).

Esse resultado confirma as conclusões de Audrestch, Keilbach e Lehmann (2006) e de Garcia (2014) ao sustentarem a tese de que o maior nível escolar de uma região e a presença de centros educacionais influenciavam no aumento da taxa de empreendedorismo, no entanto vale ressaltar que os resultados encontrados neste trabalho referem-se a uma correlação linear, não demonstrando causalidade entre as variáveis.

Quanto ao comportamento da variável saúde quando relacionada ao empreendedorismo houve grande variação nos resultados. Municípios como Araçatuba e Bauru apresentaram intensa correlação negativa, -093 e -0,90 respectivamente, o que significa que quando a taxa de empreendedorismo bruto aumenta o índice Firjan de saúde tende a diminuir. No entanto, foram encontradas correlações positivas significativas $(>0,70)$ em $44 \%$ dos municípios.

Tabela 2: Resumo das correlações entre Empreendedorismo e Desenvolvimento Socioeconômico (período 2005-2013)

\begin{tabular}{llllll}
\hline \hline Município & \multirow{2}{*}{$\begin{array}{l}\text { TEB x } \\
\text { IFDM }\end{array}$} & $\begin{array}{l}\text { TEB } \\
\text { Educação }\end{array}$ & $\begin{array}{l}\text { TEB } \\
\text { Saúde }\end{array}$ & $\begin{array}{l}\text { TEB } \\
\text { Emprego } \\
\text { Renda }\end{array}$ & $\begin{array}{c}\mathbf{X} \\
\mathbf{e}\end{array}$ \\
\hline Franca & 0,927 & 0,988 & 0,637 & 0,729 \\
Registro & 0,902 & 0,922 & 0,918 & 0,707 \\
Barretos & 0,891 & 0,940 & 0,096 & 0,315 \\
Santos & 0,880 & 0,878 & 0,906 & 0,657 \\
Pres. Prudente & 0,880 & 0,702 & 0,979 & 0,572 \\
Marília & 0,864 & 0,920 & 0,748 & 0,588 \\
Jundiaí & 0,833 & 0,905 & 0,864 & 0,070 \\
SJ do Rio Preto & 0,817 & 0,900 & 0,851 & 0,417 \\
Sorocaba & 0,801 & 0,951 & $-0,285$ & 0,358 \\
Campinas & 0,795 & 0,949 & $-0,011$ & 0,334 \\
São Paulo & 0,788 & 0,848 & 0,915 & 0,330 \\
Piracicaba & 0,771 & 0,954 & 0,174 & 0,440 \\
Bauru & 0,750 & 0,798 & $-0,902$ & 0,818 \\
São Carlos & 0,746 & 0,856 & 0,747 & $-0,356$ \\
Araçatuba & 0,663 & 0,672 & $-0,934$ & 0,755 \\
Ribeirão Preto & 0,645 & 0,901 & $-0,549$ & 0,379 \\
SJ dos Campos & 0,085 & 0,924 & $-0,075$ & $-0,794$ \\
Araraquara & $-0,137$ & 0,713 & $-0,055$ & $-0,448$ \\
\hline
\end{tabular}

Fonte: dados da pesquisa.

Assim como a variável saúde, a correlação entre empreendedorismo e emprego e renda variou bastante. São José dos Campos, Araraquara e São Carlos apresentaram correlação negativa, $-0,79 ;-0,45$ e - 0,36 respectivamente, enquanto 4 municípios (Bauru, Araçatuba, Franca e Registro) apresentaram correlação positiva superior a 0,7. Diferente do que Baptista, Escaria e Madruga (2008) encontraram, a variável emprego e renda não mostrou correlação positiva significativa em todos os municípios estudados, encontrando inclusive correlações negativas superiores a $-0,7$.

Para concluir as discussões, foram analisadas a série histórica da taxa de empreendedorismo bruto calculada para os municípios (Apêndice A) e a série histórica do índice FIRJAN de Desenvolvimento Municipal (Apêndice A). Interessante que durante esse período crises econômicas impactaram a economia nacional, como o período de 2008-2009 e, mesmo assim, houve um crescimento positivo tanto nos indicadores socioeconômicos como na taxa de empreendedorismo bruto.

Os quadros apresentados nos Apêndices A e B trazem em azul os três resultados mais positivos (melhores) de cada ano e em vermelho os três mais negativos (piores) dentre os municípios que compõem a amostra. 
Ao analisar o Apêndice A, observa-se também um resultado positivo no período de forma que houve um avanço médio de $171 \%$ no número de empresas nascentes a cada 100 habitantes, passando de uma taxa de média de 4,7 empresas em 2005 para 12,6 em 2013. Observa que Ribeirão Preto e São José do Rio Preto, ambas da região norte do estado, foram municípios que figuraram no topo do ranking durante todo o período. No fim do ranking, cidades como Araçatuba, São José dos Campos e Registro foram as mais frequentes.

Em relação aos dados do Apêndice B, Araraquara, São José do Rio Preto e Jundiaí destacamse como melhores índices ao longo do período e, ocupando as piores posições, as cidades que apresentaram os menores IFDM no mesmo período ao longo dos anos foram Araçatuba, São José dos Campos e Registro.

Por fim, relacionando os dois quadros, nota-se que São José do Rio Preto ocupa as melhores posições tanto na taxa de empreendedorismo quanto no IFDM, o que também ocorre as cidades de menores índices, de forma que Araçatuba, São José dos Campos e Registro ocupam as posições mais baixas em ambos os índices: empreendedorismo e desenvolvimento socioeconômico.

\section{Discussão dos resultados}

Os resultados apontaram a correlação positiva entre empreendedorismo e desenvolvimento socioeconômico, e também entre empreendedorismo e educação em todos os municípios pesquisados corroborando Garcia (2004) e Audretsch, Belitski e Desai, 2015. A correlação positiva entre empreendedorismo e saúde foi encontrada na maior parte dos municípios, enquanto que a correlação entre empreendedorismo e renda foi a variável que demonstrou menor correlação com empreendedorismo.

Trata-se de um estudo inédito no estado de São Paulo, utilizando a Taxa de Empreendedorismo Bruto (TEB) e a análise individual de cada município. Os resultados corroboram os resultados encontrados em outros estudos realizados no Brasil (CANEVER ET AL, 2010; FELIX; FARAH JÚNIOR, 2013) confirmando a existência de correlação entre o empreendedorismo e desenvolvimento socioeconômico, além de contribuir para o preenchimento da lacuna da carência de estudos de empreendedorismo em países em desenvolvimento (BRUTON; AHLSTROM; OBLOJ, 2008), especificamente no Brasil. Por outro lado, a variável emprego e renda mostrou resultados bastante diversos, sendo necessários outros estudos para determinar a correlação em níveis ótimos para o empreendedorismo.

\section{Considerações finais, limitações e trabalhos futuros}

Este trabalho teve como objetivo verificar a correlação entre o empreendedorismo e o desenvolvimento socioeconômico das principais cidades do estado de São Paulo por meio da comparação da taxa de empreendedorismo bruto ( $\mathrm{n}^{\mathrm{o}}$ de novos negócios criados em um período $\mathrm{n}$ a cada 1000 habitantes) com o Índice Firjan de Desenvolvimento Municipal (IFDM) no período de 2005 a 2013.

Os resultados evidenciam que o empreendedorismo é um importante fator para o desenvolvimento social e econômico, assim, conclui-se que maior atenção deve ser dirigida para seu estímulo, sendo uma forma de contribuir para o desenvolvimento local e sua qualidade de vida. Dessa forma, este estudo contribui não apenas para avançar o conhecimento escasso no Brasil sobre empreendedorismo e desenvolvimento, como também, para indicar caminhos para políticas públicas que considerem esses dois temas conjuntamente.

Novos estudos podem ser realizados para explorar como estas correlações entre empreendedorismo e desenvolvimento local ocorrem de forma a otimizar a alocação de recursos para maximização dos resultados. Outros estudos podem ser realizados abrangendo maior número de municípios do estado de São Paulo, considerando também seu porte e sua longevidade. Estudos comparativos entre os diversos estados brasileiros também são importantes para verificar se a correlações encontradas têm o mesmo comportamento em todo o país.

A principal limitação do trabalho é o fato de se constituir uma pesquisa de correlação linear, portanto os resultados não significam causalidade. Por esse motivo, novos estudos que identificassem a relação causal entre as variáveis seriam relevantes, conforme também apontam Felix e Farah Junior (2013). 
Finalmente, sugere-se como perspectiva de trabalho futuro, o estudo da influência das diferentes culturas na atividade empreendedora e o papel da cultura empreendedora no desenvolvimento regional (FRITSCH; WYRWICH, 2017).

\section{Referências}

ACS, Z. J.; DESAI, S.; HESSELS, J. Entrepreneurship, economic development and institutions. Small Business Economics, v. 31, n. 3, p. 219-234, 2008.

ADUSEI, M. Does entrepreneurship promote economic growth in Africa? African Development Review, v. 28, n. 2, p. 201-214, 2016.

AMOROS, J. E.; BORRAZ , F.; VEIGA, L. Entrepreneurship and socioeconomic indicators in Latin America. Latin American Research Review, v.51, n. 4, p. 186-201, 2016.

AUDRETSCH, D. B.; BELITSKI, M.; DESAI, S. Entrepreneurship and economic development in cities. The Annalsof Regional Science, v. 55, n. 1, p. 33-60, 2015.

AUDRETSCH, D. B.; KEILBACH, M. C.; LEHMANN E. E. Entrepreneurship and economic growth. United States: Oxford University Press, 2006, 227 p.

BAPTISTA, R., ESCARIA, V.; MADRUGA, P. Entrepreneurship, regional development and job creation: the case of Portugal. Small Business Economics, v.30, ed. 1, p. 49-58, 2008.

BOSMA, N. STERNBERG, R. Entrepreneurship as an urban event? Empirical evidence from European cities. Journal Regional Studies, v.48, n.6, p.1016-1033, 2014

BRUTON, G., D.; AHLSTROM, D.; OBLOJ, K. Entrepreneurship in emerging economies: where are we today and where should there search go in the future. Entrepreneurship Theory and Practice, v. 32, n. 1, p. 1-14, 2008.

CANEVER, M. D. et al. Entrepreneurship in the Rio Grande do Sul, Brazil: the determinants and consequences for the municipal development. Revista de Economia e Sociologia Rural, v.48, n.1, p. 85-108, 2010.

CHEN, C. C. Entrepreneurship, economic growth, and employment: a case study of Taiwan. Hitotsubashi Journal of Economics, v. 55, p. 71-88, 2014.

DVOULETÝ, O. Can policy makers count with positive impact of entrepreneurship on economic development of the Czech regions? Journal of Entrepreneurship in Emerging Economies, v. 9, n. 3, p.286-299, 2017.

FELIX, R. D. C.; FARAH JUNIOR, M. F. F. Empreendedorismo e desenvolvimento nos municípios paranaenses: uma análise dos indicadores recentes. Revista Brasileira de Planejamento e Desenvolvimento, v.2, n.2, p.104-117, 2013

FIRJAN. Relatório IFDM 2015 Índice Firjan de Desenvolvimento Municipal: Ano base 2013. Rio de Janeiro, 2015. Disponível em: http://publicacoes.firjan.org.br/ifdm/2015/\#1/z. Acesso em: $02 / 01 / 2017$.

FIRJAN. Índice FIRJAN de Desenvolvimento Municipal (IFDM). 2016. Disponível em: http://www.firjan.com.br/ifdm/consulta-ao-indice/. Acesso em: 23/12/2016.

FONTELE, R.E.S. Empreendedorismo, competitividade e crescimento econômico: evidências empíricas. Revista de Administração Contemporânea, v.14, n.6, p. 1094-1112, 2010.

FREDERICK, H.; MONSEN, E. New Zealand's perfect storm of entrepreneurship and economic development. Small Business Economics, v.37, n.2, p.187-204, 2011. 
FRITSCH, M; MUELLER, P. The effects of new business formationon regional development over time. Regional Studies, v.38, n.8, p.961-975, 2004.

FRITSCH, M.; WYRWICH, M. The effect of entrepreneurship on economic development-an empirical analysis using regional entrepreneurship culture. Journal of Economic Geography, v. 17, n.1, p. 157-189, 2017.

GARCIA, A. B. Analyzing the determinants of entrepreneurship in European cities. Small Business Economics, v.42, n.1, p.77-98, 2014

HE, X.; CHEN X. Empirical effects of entrepreneurship on regional development: a Chinese local perspective. Journal of Developmental Entrepreneurship, v.21, n.1, 2016.

JUCESP. Jucesp Online: pesquisa de empresas. 2016 . Disponível em: https://www.jucesponline.sp.gov.br/BuscaAvancada.aspx Acesso em: 18/12/2016.

KASSEEAH, H. Investigating the impact of entrepreneurship on economic development:a regional analysis. Journal of Small Business and Enterprise Development, v. 23, n. 3,p.896-916, 2016.

LI, Z.; DING, T.; LI, J. Entrepreneurship and economic development in China: evidence from a timevarying parameters stochastic volatility vector autoregressive model. Technology Analysis \& Strategic Management, v.27, n.6, p.660-674, 2015.

MUELLER, P.; VAN STEL, A.; STOREY, D. J. The effects of new firm formation on regional development over time: the case of Great Britain. Small Business Economics, v.30, n.1, p.59-71, 2008.

PRIEGER, J. E. et al. Economic Growth and the optmal level of entrepreneurship. World Development, v. 82, p. 95-109, 2016.

SCHUMPETER, J. A. Teoria do desenvolvimento econômico: uma investigação sobre lucros, capital, crédito, juro e o ciclo econômico. Tradução de Maria Silvia Possas. São Paulo: Nova Cultural, 1997, 225 p.

SI, S. et al. Entrepreneurship and poverty reduction: A case study of Yiwu, China. Asia Pacific Journal of Management, v.32, n.1, p.119-143, 2015.

SOUZA, E. C. L.; LOPES JUNIOR, G. S. Empreendedorismo e desenvolvimento: uma relação em aberto. Revista de Administração e Inovação, v. 8, n.3, p. 120-140, 2011.

STEVENSON, W. J. Estatística aplicada à administração. São Paulo: HARBRA, 2001, 495P.

TOMA, S. G.; GRIGORE, A. M.; MARINESCU, P.; Economic development and entrepreneurship. Procedia Economics and Finance, vol.8, p.436-443, 2014. 
APÊNDICE A: Série histórica da Taxa de Empreendedorismo Bruto (TEB) das cidades-sedes das Regiões Administrativas e Aglomerados Urbanos do Estado de São Paulo $(2005-2013)$

\begin{tabular}{|c|c|c|c|c|c|c|c|c|c|c|c|c|c|c|c|c|c|c|}
\hline Posição & \multicolumn{2}{|l|}{2005} & \multicolumn{2}{|l|}{2006} & \multicolumn{2}{|l|}{2007} & \multicolumn{2}{|l|}{2008} & \multicolumn{2}{|c|}{2009} & \multicolumn{2}{|l|}{2010} & \multicolumn{2}{|l|}{2011} & \multicolumn{2}{|l|}{2012} & \multicolumn{2}{|l|}{2013} \\
\hline $1^{0}$ & SJ do Rio Preto & 6,59 & SJ do Rio Preto & 6,19 & SJ do Rio Preto & 6,30 & SJ do Rio Preto & 6,86 & SJ do Rio Preto & 8,12 & Ribeirão Preto & 11,82 & São Paulo & 14,79 & Ribeirão Preto & 15,05 & Ribeirão Preto & 15,69 \\
\hline $2^{0}$ & Araçatuba & 6,02 & Ribeirão Preto & 5,35 & Ribeirão Preto & 5,67 & Ribeirão Preto & 6,66 & Bauru & 7,24 & São Paulo & 10,46 & Ribeirão Preto & 14,18 & São Paulo & 13,95 & Sorocaba & 15,24 \\
\hline 3응 & Ribeirão Preto & 5,54 & São Carlos & 5,01 & São Paulo & 5,32 & São Paulo & 6,12 & Sorocaba & 7,24 & SJ do Rio Preto & 10,36 & Campinas & 11,97 & SJ do Rio Preto & 13,26 & São Paulo & 14,21 \\
\hline $4^{0}$ & inca & 5,50 & Araçatuba & 4,94 & São Carlos & 5,29 & Araçatuba & 5,58 & Ribeirão Preto & 7,22 & Campinas & 10,26 & & 11,81 & & 12,95 & iio Preto & 14,16 \\
\hline 5o & Araraquara & 4,81 & São Paulo & 4,87 & Araçatuba & 5,17 & Sorocaba & 5,56 & Marília & 7,10 & Franca & 9,85 & Franca & 11,62 & Araçatuba & 12,66 & Araçatuba & 13,87 \\
\hline $6^{\circ}$ & Santos & 4,80 & Sorocaba & 4,54 & Sorocaba & 4,92 & Marília & 5,49 & São Paulo & 7,09 & Sorocaba & 9,83 & SJ do Rio Preto & 11,59 & São Carlos & 12,57 & Campinas & 13,48 \\
\hline $7^{0}$ & & 4,76 & Franca & & Bauru & 4,88 & & & & 7,07 & & 9,34 & & 11,55 & & 12,41 & arlos & 13,41 \\
\hline $8^{\circ}$ & São Carlos & 4,70 & Santos & 4,39 & Franca & 4,85 & São Carlos & 5,33 & Campinas & 6,98 & Marília & 9,12 & Carlos & 11,35 & & 12,36 & & 13,40 \\
\hline 90 & Sorocaba & 4,67 & Campinas & 4,24 & Marília & 4,80 & Campinas & 5,18 & Pres. Prudente & 6,75 & São Carlos & 8,84 & Bat & 10,91 & Campinas & 12,24 & Franca & 12,89 \\
\hline $10^{\circ}$ & São Paulo & 4,66 & Pres. Prudente & 4,23 & Santos & 4,71 & Bauru & 5,11 & São Carlos & 6,63 & & 8,83 & & 10,27 & caba & 11,34 & & 12,36 \\
\hline $11^{\circ}$ & & 4,48 & & 4,07 & Campinas & 4,56 & & 5,04 & & 6,5 & quara & 8,79 & Prudente & 10,11 & & 11,14 & Prudente & 12,31 \\
\hline $12^{\circ}$ & Piracicaba & 4,30 & Jundiaí & 3,98 & Pres. Prudente & 4,47 & Pres. Prudente & 4,80 & Registro & 6,49 & Registro & 8,53 & uara & 10,04 & Prudente & 10,74 & quara & 11,97 \\
\hline $13^{\circ}$ & Mar & 4,30 & Piracicaba & & & 4,36 & uara & 4,75 & & 6,2 & caba & & caba & & & 10,28 & & 11,15 \\
\hline $14^{\circ}$ & s. Prudente & 4,22 & Araraquara & 3,89 & Araraquara & 4,15 & Jundi & 4,64 & Araraquara & 6,19 & & 8,20 & & 9,36 & quara & 10,25 & Registro & 10,96 \\
\hline $15^{\circ}$ & ndiaí & 4,21 & Barretos & 3,86 & Jundiaí & 4,14 & Piracicaba & 4,52 & os Campos & 5,93 & Prudente & 8,17 & nalai & 9,26 & istro & 9,97 & & 10,95 \\
\hline $16^{\circ}$ & Barretos & 3,85 & Marília & 3,76 & Piracicaba & 3,74 & & 4,38 & & 5,92 & & 7,84 & & 8,5 & & 9,93 & Piracicaba & 10,86 \\
\hline $17^{\circ}$ & SJ dos Campos & 3,67 & SJ dos Campos & 3,34 & SJ dos Campos & 3,67 & Campos & 4,25 & Piracicaba & 5,66 & & 7,82 & & 8,54 & & 9,67 & pos & 10,10 \\
\hline & & & & 287 & & 3,29 & & 3.65 & & 4.96 & & 7.27 & & 790 & & & & 9.68 \\
\hline
\end{tabular}

Fonte: dados da pesquisa. JUCESP (2016); SEADE (2016)

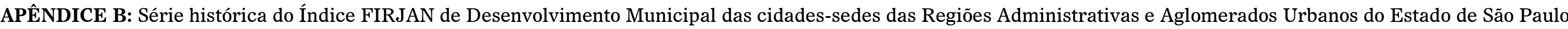
(2005-2013)

\begin{tabular}{|c|c|c|c|c|c|c|c|c|c|c|c|c|c|c|c|c|c|c|}
\hline Posição & \multicolumn{2}{|l|}{2005} & \multicolumn{2}{|l|}{2006} & \multicolumn{2}{|l|}{2007} & \multicolumn{2}{|l|}{2008} & \multicolumn{2}{|l|}{2009} & \multicolumn{2}{|l|}{2010} & \multicolumn{2}{|l|}{2011} & \multicolumn{2}{|l|}{2012} & \multicolumn{2}{|l|}{2013} \\
\hline $1^{\circ}$ & Araraquara & 0,875 & Araraquara & 0,893 & Araraquara & 0,892 & Araraquara & 0,881 & Araraquara & 0,892 & SJ do Rio Preto & 0,912 & SJ do Rio Preto & 0,916 & SJ do Rio Preto & 0,914 & SJ do Rio Preto & 0,905 \\
\hline $2^{0}$ & Bauru & 0,848 & SJ do Rio Preto & 0,870 & Jundiaí & 0,876 & SJ do Rio Preto & 0,879 & SJ do Rio Preto & 0,887 & Marília & 0,884 & Santos & 0,902 & Araraquara & 0,898 & Jundiaí & 0,889 \\
\hline $3^{\circ}$ & SJ do Rio Preto & 0,847 & Ribeirão Preto & 0,856 & SJ do Rio Preto & 0,830 & Jundiaí & 0,861 & Ribeirão Preto & 0,886 & Ribeirão Preto & 0,883 & Jundiaí & 0,895 & Jundiaí & 0,893 & Santos & 0,885 \\
\hline $4^{\circ}$ & Jundiaí & 0,840 & Bauru & 0,843 & Marília & 0,830 & Marília & 0,857 & Marília & 0,859 & Bauru & 0,878 & Bauru & 0,878 & Ribeirão Preto & 0,880 & Araraquara & 0,884 \\
\hline $5^{0}$ & Ribeirão Preto & 0,833 & Jundiaí & 0,835 & Ribeirão Preto & 0,829 & Ribeirão Preto & 0,852 & Bauru & 0,856 & Araraquara & 0,876 & Marília & 0,876 & Franca & 0,879 & Franca & 0,875 \\
\hline $6^{0}$ & São Carlos & 0,824 & Barretos & 0,832 & Bauru & 0,824 & Santos & 0,842 & São Paulo & 0,850 & Jundiaí & 0,875 & Araraquara & 0,875 & Bauru & 0,878 & Marília & 0,873 \\
\hline $7^{0}$ & Barretos & 0,820 & Marília & 0,830 & Campinas & 0,816 & Bauru & 0,842 & São Carlos & 0,849 & Campinas & 0,870 & Campinas & 0,870 & Marília & 0,877 & Ribeirão Preto & 0,872 \\
\hline $8^{\circ}$ & Campinas & 0,815 & São Carlos & 0,827 & Sorocaba & 0,802 & São Paulo & 0,838 & Piracicaba & 0,848 & Santos & 0,868 & Ribeirão Preto & 0,868 & Santos & 0,864 & São Carlos & 0,870 \\
\hline 9o & Marília & 0,814 & Campinas & 0,826 & Barretos & 0,799 & Sorocaba & 0,837 & Santos & 0,844 & Franca & 0,863 & & 0,865 & Campinas & 0,862 & Barretos & \\
\hline $10^{\circ}$ & Sorocaba & 0,808 & Sorocaba & 0,820 & São Carlos & 0,799 & Campinas & 0,836 & Jundiaí & 0,844 & Sorocaba & 0,856 & São Paulo & 0,864 & Barretos & 0,858 & Bauru & 0,858 \\
\hline $11^{\circ}$ & Santos & 0,789 & São Paulo & 0,810 & Santos & 0,794 & Piracicaba & 0,834 & Campinas & 0,843 & Piracicaba & 0,852 & Piracicaba & 0,861 & São Carlos & 0,856 & Pres. Prudente & 0,855 \\
\hline $12^{\circ}$ & Pres. Prudente & 0,787 & Araçatuba & 0,810 & São Paulo & 0,791 & São Carlos & 0,829 & Barretos & 0,837 & São Paulo & 0,851 & Barretos & 0,853 & Piracicaba & 0,855 & Piracicaba & 0,855 \\
\hline $14^{\circ}$ & Araçatuba & 0,782 & Franca & 0,800 & SJ dos Campos & 0,786 & Pres. Prudente & 0,813 & Franca & 0,820 & Barretos & 0,844 & Pres. Prudente & 0,837 & Sorocaba & 0,850 & São Paulo & 0,849 \\
\hline $15^{\circ}$ & São Paulo & 0,782 & Pres. Prudente & 0,799 & Piracicaba & 0,771 & Franca & 0,807 & Pres. Prudente & 0,814 & Araçatuba & 0,838 & São Carlos & 0,832 & s. Prudente & 0,828 & Campinas & 0,842 \\
\hline $16^{\circ}$ & Franca & 0,772 & $\begin{array}{l}\text { Piracicaba } \\
\text { Pa }\end{array}$ & 0,797 & Pres. Prudente & 0,767 & Araçatuba & 0,804 & Sorocaba & 0,812 & Pres. Prudente & 0,828 & Araçatuba & 0,822 & Araçatuba & 0,825 & Araçatuba & 0,830 \\
\hline $17^{\circ}$ & Piracicaba & 0,757 & Santos & 0,794 & Araçatuba & 0,766 & SJ dos Campos & 0,802 & SJ dos Campos & 0,809 & Registro & 0,809 & $\begin{array}{l}\text { Registro } \\
\text { Re }\end{array}$ & 0,815 & Registro & 0,822 & SJ dos Campos & 0,806 \\
\hline $18^{\circ}$ & Registro & 0,692 & Registro & 0,708 & Registro & 0,683 & Registro & 0,751 & Registro & 0,786 & SJ dos Campos & 0,797 & SJ dos Campos & 0,797 & SJ dos Campos & 0,787 & $\begin{array}{l}\text { Registro } \\
\text { R }\end{array}$ & 0,796 \\
\hline
\end{tabular}

